\title{
GENOMICS
}

\section{Polymer model predicts chromatin folding}

3C-based methods and polymer modeling indentify the impact of structural variants.

$\mathrm{T}$ he $3 \mathrm{D}$ organization of eukaryotic chromosome plays an important role in gene regulation, as the spatial proximity of regulatory elements and gene promoters governs gene activation.

The probing of genome-wide contacts with chromosome conformation capture (3C)-based techniques has led to increased understanding of genome architecture and its role in transcriptional regulation. "Yet," comments Mario Nicodemi from the Università di Napoli Federico II, in Italy, "the link between structure and function and the underlying molecular mechanisms remain to be understood." His team, together with international collaborators, including Stefan Mundlos from MPI Berlin, developed PRISMR to model chromatin folding and predict contacts between promoters and enhancers.
In contrast to methods based on $\mathrm{Hi}-\mathrm{C}$ reads and their contact matrix, PRISMR borrows the strings and binders switch (SBS) model from polymer science and applies it to chromatin conformation. The authors use genome-wide $\mathrm{Hi}-\mathrm{C}$ data in a machine learning approach to identify the minimal polymer model of a genomic locus of interest. The approach is based on a simulated annealing Monte Carlo algorithm that iteratively optimizes the polymer model to reproduce the experimental input of contact frequency matrices. For modeling of the effects of structural variants, the corresponding part of the polymer model is mutated, and then the updated model predicts, on the basis of physics only, the effects of the rearrangement on chromatin folding.

The authors used the EPHA4 locus to validate the accuracy of the model. In addition to capturing experimental observations the model also helped to identify sites of interaction with binding factors and predicted the effect of deletions and inversions that have known effects on gene expression, thus linking structure to gene misexpression.

PRISMR does not require costly computational resources and likely provides an easier way to understand the interplay between disease and spatial genome rearrangement. However, Nicodemi cautions that, when additional physical mechanisms are involved in chromatin organization, the model might need to be extended.

Lei Tang

Published online: 2 July 2018

https://doi.org/10.1038/s41592-018-0059-2

\section{Research papers}

Bianco, S. et al. Polymer physics predicts the effects of structural variants on chromatin architecture. Nat. Genet. 50, 662-667 (2018).

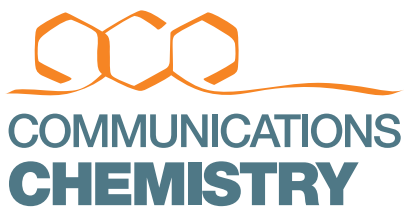

\section{NOW PUBLISHING CONTENT}

\section{A new open access journal for the chemical sciences from Nature Research}

Communications Chemistry publishes high-quality primary research articles, reviews and commentary in all areas of the chemical sciences. Papers published in the journal represent significant advances that bring new insight to a specialized area of research.

All papers are handled by experienced in-house professional editors supported by an expert Editorial board.

\section{Submit your research today and benefit from:}

- $\quad$ Thorough peer review

- Fast decision process

- High Nature editorial standards
- High visibility

- $\quad$ CC-BY open access as standard

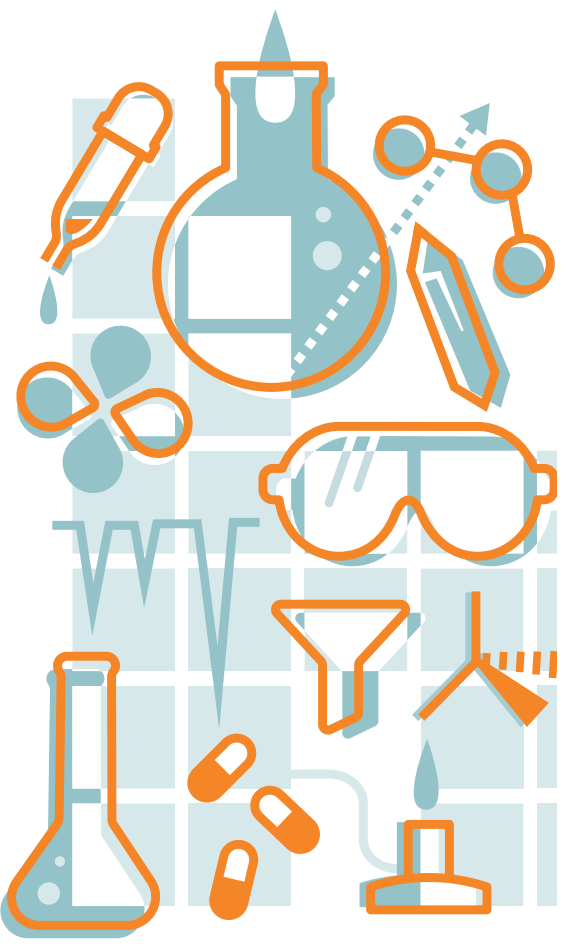

